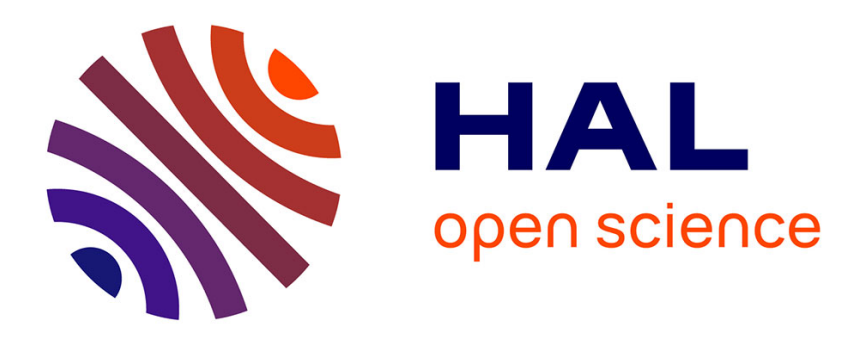

\title{
Diffusion raman spin-flip dans les semiconducteurs E. Amzallag
}

\section{- To cite this version:}

E. Amzallag. Diffusion raman spin-flip dans les semiconducteurs. Revue de Physique Appliquée, 1975, 10 (6), pp.367-377. 10.1051/rphysap:01975001006036700 . jpa-00243932

\section{HAL Id: jpa-00243932 https://hal.science/jpa-00243932}

Submitted on 1 Jan 1975

HAL is a multi-disciplinary open access archive for the deposit and dissemination of scientific research documents, whether they are published or not. The documents may come from teaching and research institutions in France or abroad, or from public or private research centers.
L'archive ouverte pluridisciplinaire HAL, est destinée au dépôt et à la diffusion de documents scientifiques de niveau recherche, publiés ou non, émanant des établissements d'enseignement et de recherche français ou étrangers, des laboratoires publics ou privés. 


\title{
DIFFUSION RAMAN SPIN-FLIP DANS LES SEMICONDUCTEURS
}

\author{
E. AMZALLAG
}

Laboratoire de Physique des Solides, associé au C. N. R. S. Université Paris VI, 4, place Jussieu, 75005 Paris, France

\begin{abstract}
Résumé. - L'étude de la diffusion Raman sur les porteurs libres d'un semiconducteur en présence d'un champ magnétique suivant le processus d'inversion du spin (spin-flip) présente un intérêt multiple : meilleure connaissance du mécanisme de diffusion lui-même, détermination du facteur $g$ des porteurs libres en rapport avec la structure de bandes du matériau étudié, enfin et surtout génération d'une émission stimulée pouvant conduire à la réalisation de sources lumineuses cohérentes et intenses, de longueur d'onde continûment accordable au moyen du champ magnétique appliqué.

Cet exposé passe en revue les principaux résultats obtenus ces dernières années, en même temps qu'il présente quelques-uns de nos propres résultats dans $\mathrm{CdS}$ pompé avec un laser à argon $(5145 \AA)$, et dans InSb pompé avec un laser à $\mathrm{CO}_{2}(10,6 \mu \mathrm{m})$.
\end{abstract}

Abstract. - The study of Raman scattering by mobile electrons in a magnetic field through the spin-flip process is of multiple interest : a better knowledge of the scattering mechanism itself, the determination of the $g$-factor for the free electrons which is related to the band structure of the material, and above all the generation of a stimulated radiation which leads to the achievement of coherent and intense sources, whose wavelength can be continuously tuned through the applied magnetic field.

This talk reviews the main results obtained during the previous years, while it presents some of our own results in CdS pumped by an Argon laser $(5145 \AA)$ and in InSb pumped by a $\mathrm{CO}_{2}$ laser $(10.6 \mu \mathrm{m})$.

1. Introduction. - La diffusion Raman par inversion de spin (spin-flip) dans les semiconducteurs connaît actuellement un développement remarquable par suite de l'intérêt qu'elle présente d'une part sur le plan fondamental avec l'étude du mécanisme de diffusion lui-même en rapport avec les propriétés physiques des électrons dans les semiconducteurs, et d'autre part sur le plan des applications avec la réalisation de sources lumineuses cohérentes et intenses accordables en longueur d'onde, qui peuvent être utilisées en spectroscopie à haute résolution et aussi en optique non linéaire.

Par rapport aux autres processus non linéaires permettant d'obtenir des sources accordables telles que la génération paramétrique [1], la génération de la somme ou la différence de deux fréquences [2], la diffusion Raman sur les polaritons [3], le processus spin-flip a l'avantage de fournir des raies beaucoup plus intenses et excessivement plus fines. De plus, il ne nécessite pas de condition critique d'accord de phase.

L'objet de cet exposé est de présenter les principes de base de cet effet de diffusion, d'illustrer ses caractéristiques essentielles à l'aide des principaux résultats obtenus ces dernières années, essentiellement sur InSb puisqu'il constitue le matériau idéal actuel, et enfin d'examiner très rapidement quelques applications immédiates.
2. Principes de base. - Le processus Raman spinflip implique une excitation par laquelle le spin d'un électron de conduction subit une inversion par rapport à un champ magnétique extérieur. Les relations de conservation de l'énergie et du moment s'écrivent :

$$
\begin{aligned}
& \hbar \omega_{\mathrm{d}}=\hbar \omega_{0} \pm \hbar \omega_{\mathrm{e}} \\
& \mathbf{k}_{\mathrm{d}}=\mathbf{k}_{\mathbf{0}} \pm \mathbf{q}_{\mathrm{e}}
\end{aligned}
$$

où $\hbar \omega_{\mathrm{d}}, \hbar \omega_{0}$ et $\hbar \omega_{\mathrm{e}}$ sont respectivement les énergies du photon diffusé, du photon incident et de l'excitation, $\mathbf{k}_{\mathrm{d}}, \mathbf{k}_{0}$ et $\mathbf{q}_{\mathrm{e}}$ sont les vecteurs d'onde correspondants. Le signe - s'applique à la diffusion Stokes et le signe + à la diffusion anti-Stokes. La longueur d'onde de la lumière diffusée est accordable au moyen du champ magnétique, puisque l'on a :

$$
\hbar \omega_{\mathrm{e}}=g^{*} \beta H
$$

où $\beta$ est le magnéton de $\mathrm{Bohr}=e \hbar / 2 m c, g^{*}$ est le facteur gyromagnétique de l'électron de conduction et $H$ le champ magnétique. Par ailleurs, l'excitation spinflip n'étant pas dispersive, l'éq. (2) est facilement satisfaite, sans condition sévère d'accord de phase.

2.1 Diffusion SPONTANÉE. - La diffusion Raman est un processus de second ordre auquel on peut faire correspondre une probabilité de transition proportion- 
nelle à $|R|^{2}$, où $R$ est l'élément de matrice donné par [4]:

$$
\begin{aligned}
R=\sum_{t}\left\{\frac{\left\langle f\left|\varepsilon_{\mathrm{d}}^{*} \mathbf{v}\right| t\right\rangle\left\langle t\left|\varepsilon_{0} \cdot \mathbf{v}\right| i\right\rangle}{\hbar \omega_{0}-\left(E_{t}-E_{i}\right)}-\right. \\
\left.-\frac{\left\langle f\left|\varepsilon_{0} \cdot \mathbf{v}\right| t><t\left|\varepsilon_{\mathrm{d}}^{*} \cdot \mathbf{v}\right| i\right\rangle}{\hbar \omega_{\mathrm{d}}+\left(E_{t}-E_{i}\right)}\right\} .
\end{aligned}
$$

Le système est initialement dans l'état $|i\rangle$ d'énergie $E_{i}$, il est finalement dans l'état $|f\rangle$ d'énergie $E_{f}$. La sommation porte sur tous les états intermédiaires $\mid t>$. La transition $|i\rangle \rightarrow|t\rangle$ est virtuelle. Elle ne nécessite pas de conservation d'énergie. $\varepsilon_{0}$ et $\varepsilon_{d}$ sont les vecteurs polarisations des photons incidents et diffusés. $\mathbf{v}$ est l'opérateur vitesse donné dans le cas présent par

$$
m \mathbf{v}=-i h \boldsymbol{\nabla}+\frac{e \mathbf{A}}{c}
$$

où $\mathbf{A}$ est le potentiel-vecteur dont dérive le champ magnétique.

A cette diffusion correspond une section efficace différentielle donnée par [5]

$$
\frac{\mathrm{d} \sigma}{\mathrm{d} \Omega}=\left(\frac{e^{2}}{m c^{2}}\right)^{2}\left(\frac{\omega_{\mathrm{d}}}{\omega_{0}}\right)^{2} \frac{n_{\mathrm{d}}}{n_{0}} m^{2} \sum_{i}|R|^{2}
$$

où $n$ désigne l'indice de réfraction du matériau et où la sommation porte sur tous les états initiaux qui peuvent contribuer à la diffusion. L'expression (5) se rapporte en fait à l'effet spontané où la section efficace est indépendante des densités de photons incidents et diffusés. En dehors de cette section efficace, l'effet spontané est aussi caractérisé par la largeur de raie (largeur à mihauteur), ces deux paramètres $\mathrm{d} \sigma / \mathrm{d} \Omega$ et $\Gamma$ jouant un rôle tout à fait fondamental dans la description du laser spin-flip.

2.2 Diffusion stimulée. - C'est évidemment l'effet stimulé qui nous intéresse ici, dans la mesure où il permet d'obtenir une émission intense et cohérente consécutive au pompage du système par la lumière incidente (pompe). Cela n'implique aucunement une inversion de population, car dans ce cas le processus Stokes verrait ses états finaux (supérieurs en énergie) bloqués et ne pourrait avoir lieu, ce qui n'est pas le cas. Bloembergen et Shen [6] ont montré que l'effet Raman stimulé peut être décrit par un coefficient optique non linéaire du $3^{\mathrm{e}}$ ordre. L'interaction du champ $\mathbf{E}_{0}$ (de la pompe) et du champ $\mathbf{E}_{d}$ (de la lumière diffusée) induit dans le matériau une polarisation non linéaire à la fréquence $\omega_{\mathrm{d}}$, donnée par

$$
P^{\mathrm{NL}}\left(\omega_{\mathrm{d}}\right)=\chi_{R}\left|E_{0}\right|^{2} E_{\mathrm{d}} .
$$

En pratique, dans le cas de la diffusion Stokes, la susceptibilité $\chi_{R}$ est purement imaginaire et négative. $\mathrm{La}$ résolution de l'équation d'onde relative à l'onde

diffusée conduit à une absorption négative, donc à un gain à la fréquence $\omega_{\mathrm{d}}$, donné par [5]

$$
g=\frac{16 \Pi^{2} c^{2}}{\hbar \omega_{\mathrm{d}}^{3} n_{\mathrm{d}}^{2}}\left(\frac{\mathrm{d} \sigma}{\mathrm{d} \Omega}\right) \frac{I_{0}}{\Gamma}
$$

où $I_{0}$ est l'intensité de la pompe, $\mathrm{d} \sigma / \mathrm{d} \Omega$ est la section efficace de l'effet spontané (éq. (5)) et $\Gamma$ la largeur de raie spontanée.

Par conséquent, pour un échantillon de longueur $l$, de coefficient d'absorption $\alpha$ et de coefficient de réflexion $R$, on obtient une oscillation de la lumière diffusée pour

$$
R \exp (g-x) l \geqslant 1 .
$$

On a alors une émission laser avec toutes les caractéristiques de cohérence, monochromaticité, etc... d'un laser conventionnel. Le tableau I montre une comparaison qualitative des processus de diffusion Raman spontanée et stimulée.

\section{TABLEAU I}

Comparaison qualitative des processus de diffusion Raman spontanée et stimulée

Diffusion spontanée

dans toutes les directions largeur de raie spontanée processus sans seuil conversion $10^{-6}$

croissance linéaire du signal

\section{Diffusion stimulée}

cohérence spatiale de la pompe largeur de raie beaucoup plus fine processus avec seuil conversion 1 à $80 \%$ théoriquement $100 \%$ croissance exponentielle du signal

On voit que la réalisation d'un laser spin-flip nécessite un gain Raman suffisamment élevé pour dépasser les pertes optiques dans la cavité constituée par l'échantillon. L'expression (7) de ce gain montre qu'il est indispensable que l'effet spontané soit lui-même relativement intense et que sa largeur de raie soit suffisamment étroite.

3. Formulation microscopique. - La physique microscopique du gain Raman est en fait incluse pour une bonne part dans l'expression (4) de l'élément de matrice de transition $R$. Le premier calcul théorique de l'effet est dû à Yafet [7] ; nous nous contenterons ici de rappeler les principaux résultats de cette étude.

Il faut préciser d'abord que dans ce processus de diffusion, les transitions sont de nature dipolaire électrique et qu'elles activent, par l'intermédiaire du processus Raman, le dipôle essentiellement magnétique associé à l'excitation spin-flip. L'effet nécessite une interaction spin-orbite appréciable, car la lumière ne se couple pas au spin directement mais par l'intermédiaire du couplage spin-orbite [8]. Précisément, une des raisons pour lesquelles InSb constitue un matériau particulièrement favorable est l'importance de son 
couplage spin-orbite $\left(\Delta \simeq 0,8 \mathrm{eV}, E_{\mathrm{g}} \simeq 0,24 \mathrm{eV}\right)$, l'autre raison essentielle étant son facteur $g^{*}$ élevé $(-50)$, qui conduit à une large gamme de fréquence accordable, donnée par $\Delta v=g^{*} \beta H / \hbar$.

Dans les semiconducteurs à faible gap comme InSb, la plus grande contribution provient des transitions interbandes. La figure 1 représente la structure de

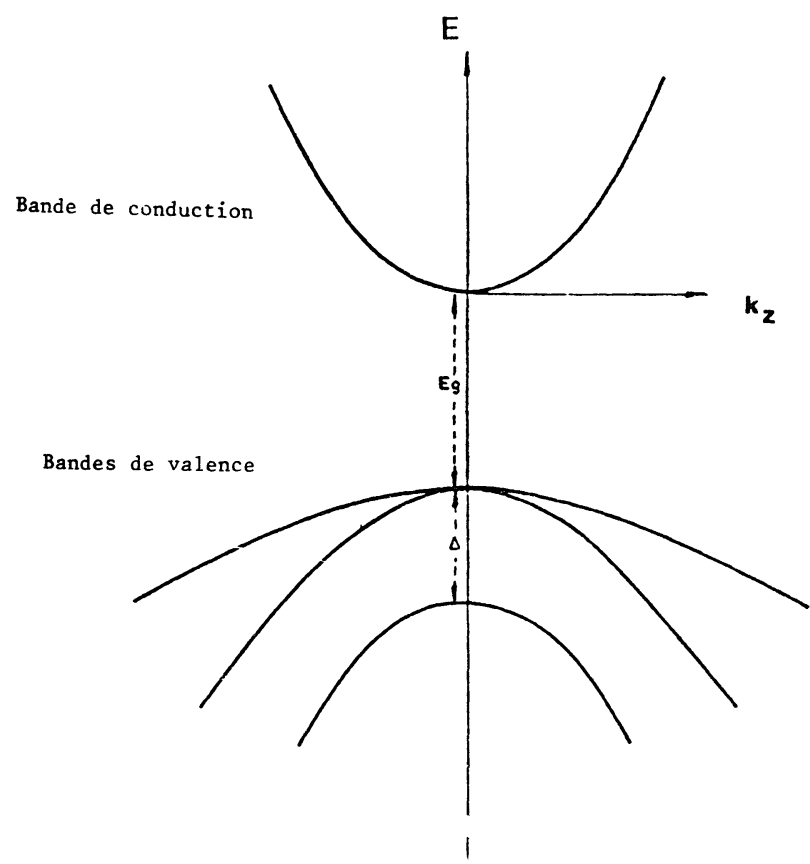

FIG. 1. - Structure de bandes de InSb au voisinage de $\mathbf{k}=0$.

bandes de $\mathrm{InSb}$ au voisinage de $\mathbf{k}=0$. On peut voir d'après les dénominateurs en énergie de l'expression (4) de $R$, que ce sont les transitions en centre de zone qui sont les plus importantes. En présence d'un champ magnétique appliqué dans la direction $\mathrm{O} z$, ces bandes se décomposent en une série de sous-bandes qui correspondent à la quantification orbitale du mouvement de l'électron dans le plan $x, y$ (niveaux de Landau), chacune de ces sous-bandes se dédoublant ensuite en deux niveaux spin $\uparrow$ et spin $\downarrow$, du fait de l'interaction spinchamp (Fig. 2). Dans le cas simple d'une bande d'énergie parabolique et isotrope, ces niveaux d'énergie sont donnés par:

$E\left(k_{z}, n, m_{\mathrm{s}}\right)=\frac{\hbar^{2} k_{z}^{2}}{2 m^{*}}+\left(n+\frac{1}{2}\right) \hbar \omega_{\mathrm{c}}+g^{*} \beta H m_{\mathrm{s}}$

où $n$ est le nombre quantique de Landau $=0,1,2, \ldots$ $\omega_{\mathrm{c}}=e H / m^{*} c$ est la fréquence cyclotron, et $m_{\mathrm{s}}$ le nombre quantique relatif à la composante suivant $\mathrm{O} z$ du moment angulaire du spin $\left(m_{\mathrm{s}}= \pm \frac{1}{2}\right)$. En fait, cette description n'est valable que pour la bande de conduction, encore faut-il tenir compte dans le cas d'un semiconducteur à faible gap comme InSb, d'une certaine non-parabolicité de la bande qu'on peut justifier par la théorie k.p de Kane, et qui conduit à des niveaux de Landau non uniformes. Pour les états dégénérés

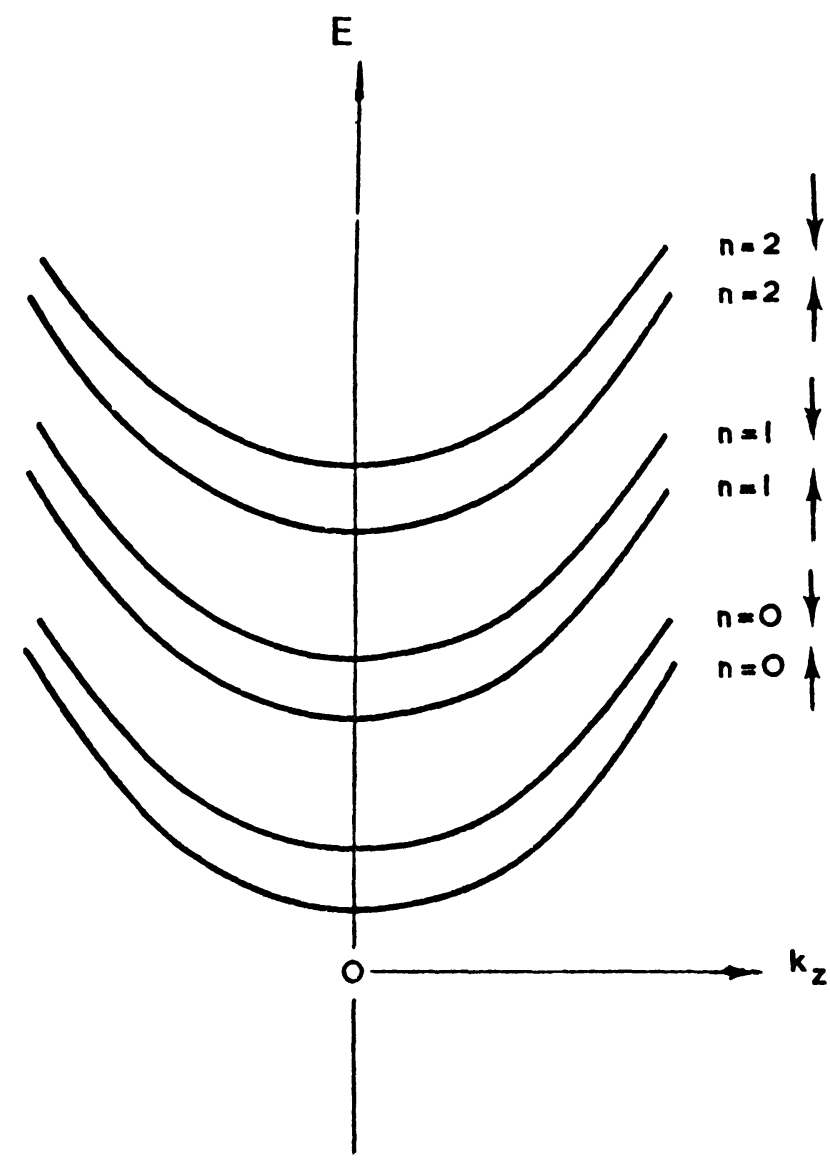

Fig. 2. - Sous-niveaux de Landau de la bande de conduction dans InSb en présence d'un champ magnétique $\mathbf{H} / / \mathbf{O z}$.

du haut de la bande de valence, la description est beaucoup plus compliquée. On obtient différentes échelles d'énergie dont les niveaux ne sont pas également espacés, sauf pour $n$ élevé.

Le processus microscopique généralement admis pour la diffusion Stokes par exemple, est un processus à 2 étapes (Fig. 3). Il implique une excitation virtuelle d'un électron d'un état de la bande de valence vers le niveau supérieur $0 \downarrow$ de la bande de conduction avec absorption du photon incident, suivie d'une transition virtuelle d'un électron du niveau inférieur $0 \uparrow$ de la bande de conduction vers l'état de la bande de valence avec émission du photon diffusé. C'est parce que certains états de la bande de valence sont mixés en spins (par exemple l'état $J=\frac{3}{2}$ et $m_{\mathrm{s}}=\frac{1}{2}$ qu'on peut représenter par une fonction d'onde de la forme

$$
\left.\psi=\left[-\sqrt{\frac{2}{3}} z \uparrow+\sqrt{\frac{1}{6}}(x+i y) \downarrow\right]\right)
$$

qu'ils peuvent servir d'états intermédiaires et que le processus spin-flip peut avoir lieu.

Le traitement de Yafet se simplifie dans le cas d'un modèle à deux bandes dans un semiconducteur à faible gap. On trouve essentiellement [8]

$$
\frac{\mathrm{d} \sigma}{\mathrm{d} \Omega} \propto\left(\frac{e^{2}}{m_{\mathrm{s}} c^{2}}\right)^{2}\left[\frac{E_{\mathrm{G}} \hbar \omega_{0}}{E_{\mathrm{G}}^{2}-\left(\hbar \omega_{0}\right)^{2}}\right]^{2}\left|\sigma . \varepsilon_{0} \varepsilon_{\mathrm{d}}\right|^{2}
$$




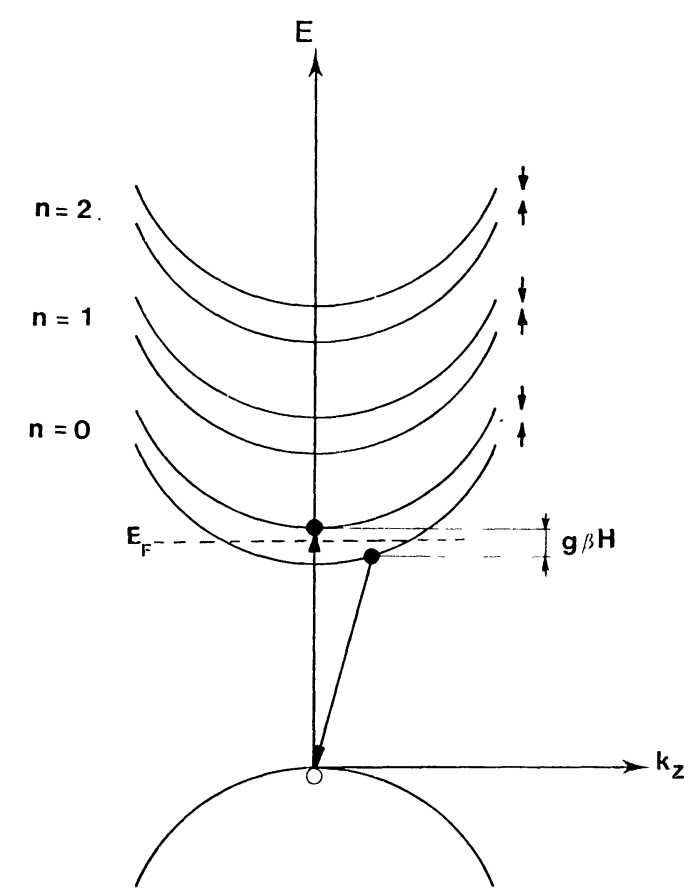

Fig. 3. - Processus de diffusion spin-flip interbande.

où $m_{\mathrm{s}}$ est la masse associée au spin

$$
\left(\frac{m}{m_{\mathrm{s}}}=\frac{\left|g^{*}\right|}{2}\right)
$$

et $\sigma$ la matrice de spin de Pauli, d'où il ressort les trois propriétés suivantes :

1) Dans les semiconducteurs à faible gap, $\left|g^{*} / 2\right| \gg 1$, la section efficace est $\left(\mathrm{m} / \mathrm{m}_{\mathrm{s}}\right)^{2}$ fois plus grande que la section efficace Thomson, soit 625 fois dans InSb.

2) Lorsque la fréquence de la pompe $\omega_{0}$ approche de la fréquence correspondant au gap $E_{\mathrm{g}}$, il y a un effet de résonance sur la section efficace qui augmente alors considérablement.

3) La lumière diffusée obéit à des règles de sélection liées à la polarisation des photons incidents.

Cependant, il convient de tenir compte de la statistique de distribution des électrons de conduction, puisqu'en vertu du principe de Pauli, il importe que l'état initial soit plein et l'état final vide. Dans le cas d'une distribution dégénérée, cela revient à multiplier la section efficace par le facteur

$$
S=f_{n \uparrow}(k)\left[1-f_{n \downarrow}(k)\right]
$$

où $f_{n \uparrow}$ et $f_{n \downarrow}$ sont les facteurs d'occupation de Fermi des deux sous-niveaux de Landau correspondant à $n \uparrow$ et $n \downarrow$. A la température limite $T=0 \mathrm{~K}, S$ n'est autre que $N_{\uparrow}-N_{\downarrow}$, la différence entre les concentrations des spins $\uparrow$ et $\downarrow$. Le calcul théorique de Wherret et Harper [9] conduit à des résultats frappants que l'on peut illustrer à l'aide des deux figures suivantes.

La figure 4 représente deux situations différentes :

a) Le niveau de Fermi est situé entre les niveaux $0 \uparrow$

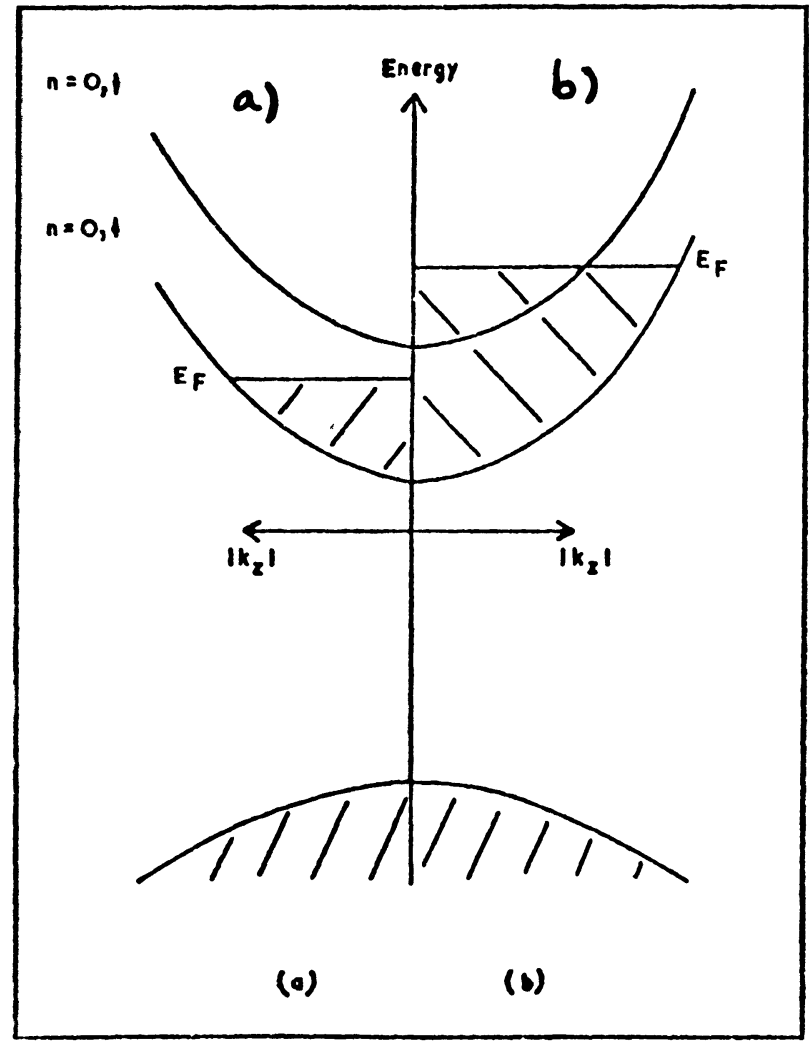

Fig. 4. - Influence de la population sur la diffusion spin-flip : a) $E_{\mathrm{F}}<h \omega_{\mathrm{sf}}$; b) $h \omega_{\mathrm{sf}}<E_{\mathrm{F}}<h \omega_{\mathrm{c}}$ (Wherret et al. [9]).

et $0 \downarrow, E_{\mathrm{F}}<\hbar \omega_{\mathrm{sf}}$, tous les électrons peuvent alors participer au processus, et on a

$$
S=N(E)=\left(\frac{2 e H}{h^{2} c}\right)\left(2 m^{*} E\right)^{1 / 2} \equiv a E^{1 / 2} .
$$

b) Le niveau de Fermi est au-delà de $0 \downarrow$, avec $\hbar \omega_{\mathrm{sf}}<E_{\mathrm{F}}<\hbar \omega_{\mathrm{c}}$, les électrons du niveau $0 \downarrow$ au nombre de $a\left(E_{\mathrm{F}}-\hbar \omega_{\mathrm{sf}}\right)^{1 / 2}$ et un nombre égal d'électrons du niveau $0 \uparrow$ ne peuvent participer, d'où

$$
S=N-2 a\left(E_{\mathrm{F}}-\hbar \omega_{\mathrm{sf}}\right)^{1 / 2} .
$$

La figure 5 donne les variations du coefficient $N \uparrow-N \downarrow$ d'une part en fonction du champ magnétique et d'autre part en fonction de la concentration des porteurs libres [5]. Ces variations traduisent le passage de $E_{\mathrm{F}}$ aux différents sous-niveaux de Landau et les singularités de densité d'états correspondantes. On voit que le cas le plus favorable est celui où $H$ est tel que $E_{\mathrm{F}}=\hbar \omega_{\text {sf }}$, c'est-à-dire quand le niveau $0 \uparrow$ est entièrement plein et le niveau $0 \downarrow$ entièrement vide (limite quantique) auquel cas $S$ est égal à la densité de porteurs $N$. Ces figures illustrent les limitations susceptibles d'intervenir en fonction du champ magnétique ou du nombre de porteurs.

Un autre facteur intervenant dans le gain Raman de l'expression (7) est la largeur de raie $\Gamma$, dont on peut dire que la formulation microscopique reste très complexe. L'analyse de la forme de raie dans InSb doit tenir compte des contributions relatives de la non- 


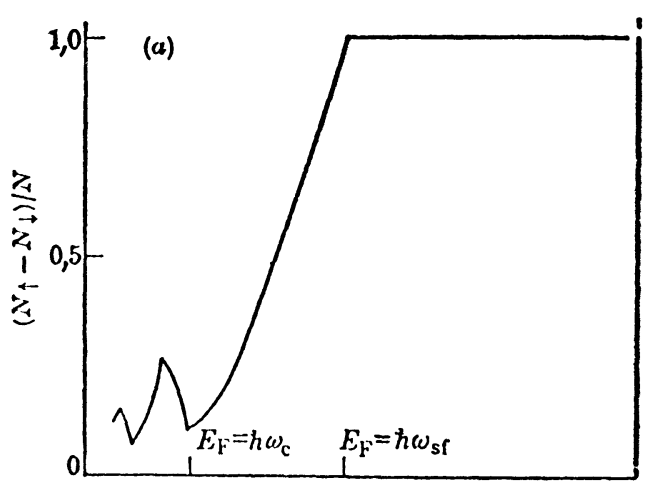

H

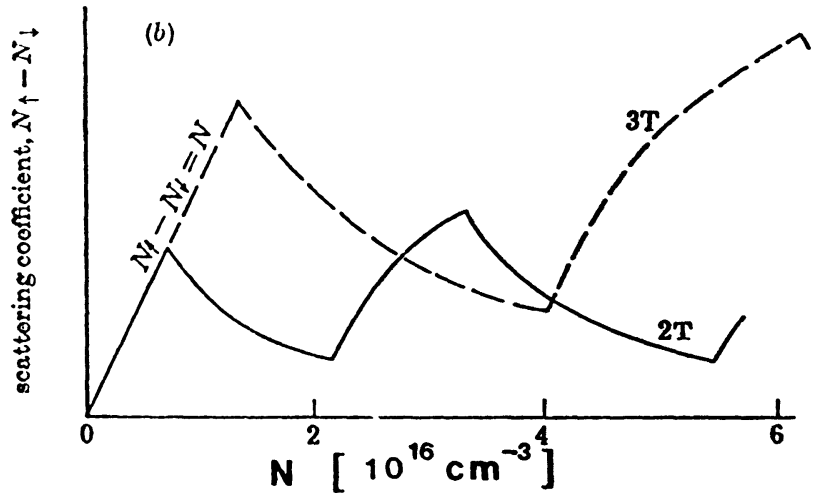

FIG. 5. - Variation du coefficient de diffusion $N \uparrow-N \downarrow$ : a) en fonction du champ magnétique, b) en fonction de la concentration des porteurs (Dennis et al. [5]).

parabolicité de la bande de conduction et de la diffusion électronique [10].

Enfin une optimisation de la cavité doit passer par une analyse des pertes de cette cavité : pertes par réflexion $(R=37 \%$ dans InSb sous incidence normale) et par absorption. Ces dernières sont dues surtout à des transitions intrabandes sur les porteurs libres, quand la fréquence de la pompe est éloignée de la limite d'absorption (InSb pompé avec un laser à $\mathrm{CO}_{2}$ par exemple) ou à des transitions interbandes lorsque la fréquence-pompe est proche de cette limite d'absorption (InSb pompé avec un laser à $\mathrm{CO}$ ). Il faut noter que pour des champs magnétiques élevés ou de fortes concentrations de porteurs, le coefficient d'absorption dû aux porteurs libres augmente considérablement [11]. Comme autres causes d'absorption, on peut citer la résonance cyclotron et ses harmoniques $\left(n \omega_{\mathrm{c}}\right)$, l'absorption sur le phonon LO et les harmoniques cyclotrons assistés du phonon LO $\left(n \omega_{\mathrm{c}}+\omega_{\mathrm{LO}}\right)$ [12]. Une dernière remarque à cet égard porte sur la configuration expérimentale. La géométrie transverse qui consiste à détecter la lumière diffusée à $90^{\circ}$ de la direction de propagation de la pompe a le désavantage que le gain Raman ne joue que sur le diamètre du filament de l'échantillon pompé effectivement ( $\sim 200 \mu$ de diamètre) alors que l'absorption a lieu sur toute l'épaisseur du cristal (quelques $\mathrm{mm}$ ). La géométrie colinéaire (où l'on détecte le faisceau diffusé dans la direction de propagation de la pompe) correspond à une meilleure optimisation de la cavité.
4. Principaux résultats expérimentaux. - Historiquement, les grandes étapes qui ont marqué l'étude de la diffusion spin-flip peuvent se résumer ainsi :

1966 : La diffusion Raman sur les porteurs libres en présence d'un champ magnétique est prévue par Wolff [13]. La théorie est étendue au processus d'inversion de spin par Yafet [7].

1967 : Première observation de l'effet spontané sur InSb par Slusher et al. [14].

1970 : Première observation de l'effet stimulé par Patel et Shaw [15]. Démonstration de l'effet résonnant et première observation de l'effet stimulé en continu (CW) par Mooradian et al. [16].

Depuis, l'étude s'est développée dans plusieurs directions : optimisation des caractéristiques de la raie spin-flip (section efficace, largeur de raie), réduction du seuil d'intensité de la pompe, accroissement de la puissance de sortie, possibilités d'application en spectroscopie à haute résolution et en optique non linéaire, en même temps que l'effet était observé dans d'autres semiconducteurs que InSb [8].

On peut illustrer les caractéristiques essentielles du laser spin-flip à l'aide des résultats expérimentaux suivants.

4. 1 EFfet SPONTANÉ. EFFET STIMUlÉ. - La figure 6 est une représentation typique de la variation de la puissance Stokes en fonction de la puissance incidente [11]. Pour de faibles puissances de la pompe, on a une dépendance linéaire qui traduit le processus spontané. Puis le signal croît de trois ordres de grandeur pratiquement, quand la puissance de la pompe dépasse un certain seuil. Il a ensuite tendance à se saturer par suite de l'affaiblissement de la puissance de la pompe dans le matériau, mais aussi et surtout à cause de la saturation du système de spins.

4.2 Saturation DES SPINS. - Elle provient essentiellement de ce que le temps de relaxation des spins est relativement trop long $\left(\tau_{\mathrm{s}} \simeq 10^{-9}\right.$ à $\left.10^{-8} \mathrm{~s}\right)$ [17], [20]. Une fois l'égalisation des populations atteinte sur les deux niveaux $0 \uparrow$ et $0 \downarrow$, les spins ne peuvent relaxer rapidement vers le niveau $0 \uparrow$, il en résulte un blocage, le gain Raman tombe à zéro en l'absence d'effets de relaxation ou de diffusion.

Cela se traduit expérimentalement par une limitation de la puissance de sortie, donc du facteur de conversion, mais aussi par une réduction de la durée de l'impulsion spin-flip (typiquement de l'ordre de 30 ns comparée à la durée de l'impulsion de la pompe $\simeq 200 \mathrm{~ns}$ ) [11].

Pour diminuer ces effets de saturation, il faut donc réduire $\tau_{\mathrm{S}}$ (introduction d'une impureté paramagnétique dans InSb [11]) ou augmenter la vitesse de diffusion des électrons, par exemple par application d'un champ électrique transverse, ce qui aurait pour effet d'assurer un renouvellement rapide des électrons dans le filament actif. 


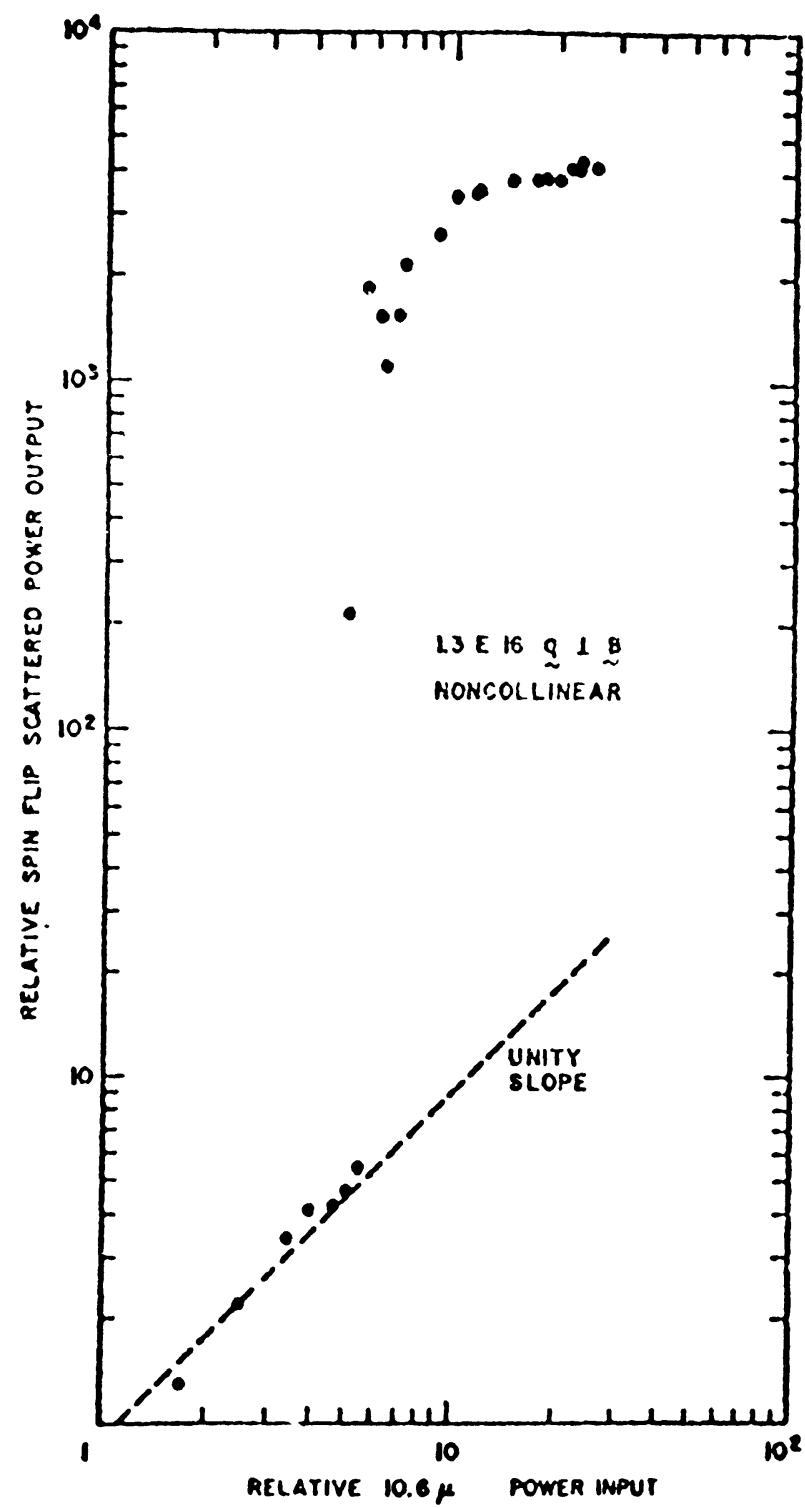

FIG. 6. - Puissance diffusée en fonction de la puissance incidente dans l'effet Raman spin-flip spontané et stimulé (Patel et al. [11]).

4.3 Effet Résonnant. Seuil. FaCteur de ConverSion. - La meilleure démonstration de l'effet résonnant réside dans l'abaissement spectaculaire du seuil de stimulation, lorsque dans InSb par exemple, on passe d'un pompage par laser à $\mathrm{CO}_{2}(10,6 \mu)$ à un pompage par un laser à $\mathrm{CO}$, c'est-à-dire à des longueurs d'onde très voisines de la limite d'absorption de $\operatorname{InSb}(5,3 \mu)$. Dans le $1^{\text {er }}$ cas, les seuils observés sont de l'ordre de $4 \times 10^{4} \mathrm{~W} / \mathrm{cm}^{2}$ [18], ce qui nécessite des'lasers à impulsion, et ce qui explique qu'un fonctionnement en continu $(\mathrm{CW})$ à $10,6 \mu$ n'ait pas été obtenu à ce jour. Dans le $2^{\mathrm{e}}$ cas, des seuils aussi bas que $40 \mathrm{~W} / \mathrm{cm}^{2}$ ont pu être observés [19], c'est-à-dire qu'on peut obtenir un effet stimulé avec un laser à CO continu, d'une puissance de $10 \mathrm{~mW}$ à peine, focalisé sur un filament de $200 \mu$ de diamètre.

Les facteurs de conversion sont limités essentielle- ment par les différents processus de saturation. A $10,6 \mu$, c'est la saturation des spins qui prédomine [20]. Les électrons du niveau $0 \uparrow$ sont disponibles à des taux de $10^{19}$ à $10^{21} \mathrm{~s}^{-1}$ comparés à des flux de photons de $10^{22}$ à $10^{24} \mathrm{~s}^{-1}$ (laser $\mathrm{CO}_{2}$ à impulsions). On obtient des facteurs de conversion $\leqslant 5 \%$ [18]. Dans le cas d'un laser CO continu, les flux de photons sont compris entre $10^{19}$ et $10^{21} \mathrm{~s}^{-1}$, de sorte que l'affaiblissement de la puissance de la pompe dans le matériau devrait jouer autant que la saturation des spins. Les facteurs de conversion fréquemment observés sont de l'ordre de $50 \%$ et peuvent même atteindre $80 \%$ [17].

4.4 Limitation en Champ magnétiQue. - A faible champ, l'effet stimulé est limité par le fait que la condition de limite quantique $E_{\mathrm{F}}=h \omega_{\mathrm{sf}} \mathrm{n}$ 'est plus satisfaite. Le champ minimum nécessaire pour obtenir une stimulation dépend évidemment du nombre de porteurs. Il est de $25 \mathrm{kG}$ environ pour un échantillon de

$$
N=10^{16} \mathrm{~cm}^{-3} \text {, }
$$

mais il peut être très bas, de l'ordre de $400 \mathrm{G}$, pour un échantillon de $N=10^{15} \mathrm{~cm}^{-3}$ (Patel [21]).

A haut champ, l'effet laser peut s'annuler par suite des pertes par absorption dans la cavité. La figure 7 montre une variation typique de l'effet stimulé en fonction du champ magnétique [12] pour un échantillon de

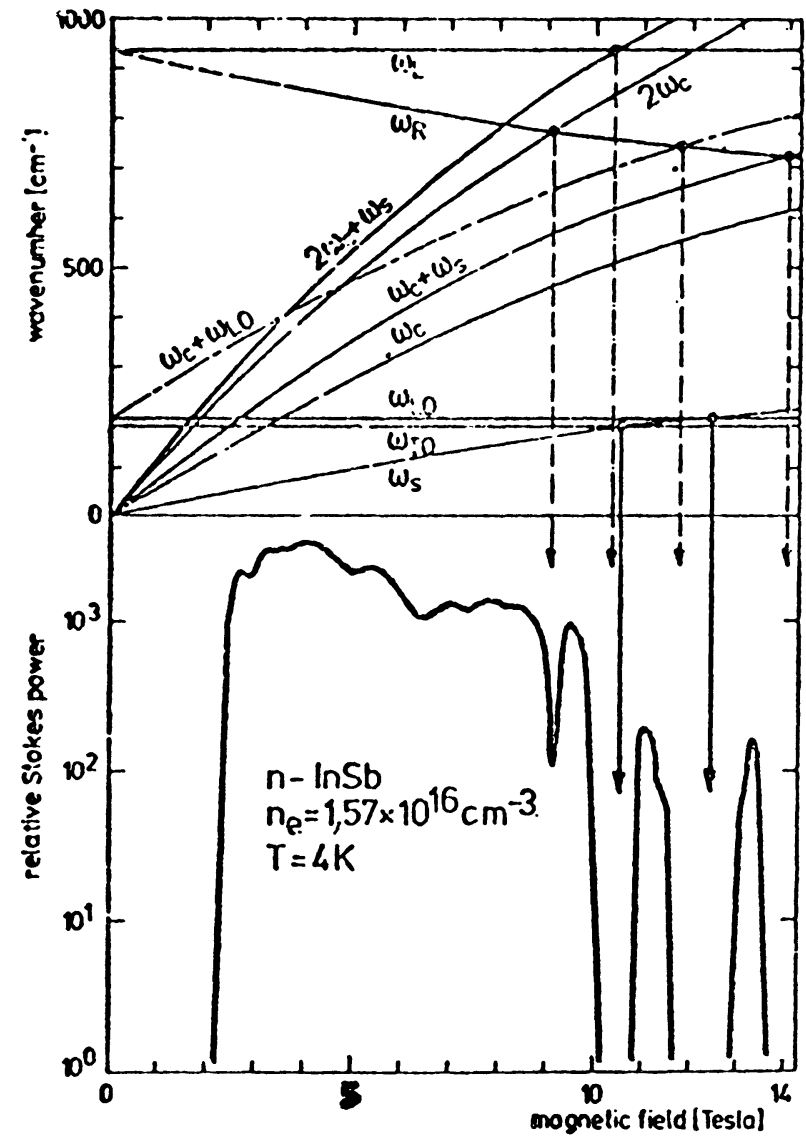

Fig. 7. - Influence de l'absorption due à la résonance cyclotron et aux phonons sur la diffusion spin-flip (Wachernig et al. [12]). 
$N=1,6 \times 10^{16} \mathrm{~cm}^{-3}$. On y voit la coupure à faible champ, et aussi plusieurs creux à champ élevé qui pourraient être dus à l'absorption suivant les transitions indiquées sur la figure.

4. 5 Polarisation. - Les règles de sélection théoriques [22] qui interviennent dans l'expression (10) de la section efficace sont généralement vérifiées par l'expérience. La configuration qui s'avère la plus efficace est finalement la géométrie colinéaire $\mathbf{k}_{0} / / \mathbf{k}_{\mathrm{d}}$, $\operatorname{avec} \mathbf{E}_{0} / / \mathbf{H}$ et $\mathbf{E}_{\mathrm{d}} \perp \mathbf{H}$.

4.6 ANTI-STOKes ET STOKES D'ORDRE SUPÉRIEUR. Théoriquement, la diffusion Raman stimulée à la fréquence anti-Stokes n'est pas possible. En effet, la polarisation correspondante, analogue à celle de l'expression (6), conduit à un gain négatif, c'est-à-dire à une absorption, à moins d'une inversion de population provoquée, entre les états $0 \uparrow$ et $0 \downarrow$. L'émission stimulée A-S observée expérimentalement est décrite plutôt à l'aide d'une interaction paramétrique couplant les champs Stokes et anti-Stokes et nécessitant des conditions d'accord de phase (la configuration colinéaire est favorable dans InSb).

La diffusion double-Stokes implique aussi des couplages paramétriques entre les différents champs, mais également le processus répétitif où la première Stokes sert de pompe pour engendrer la deuxième, la deuxième pour engendrer la troisième, etc...

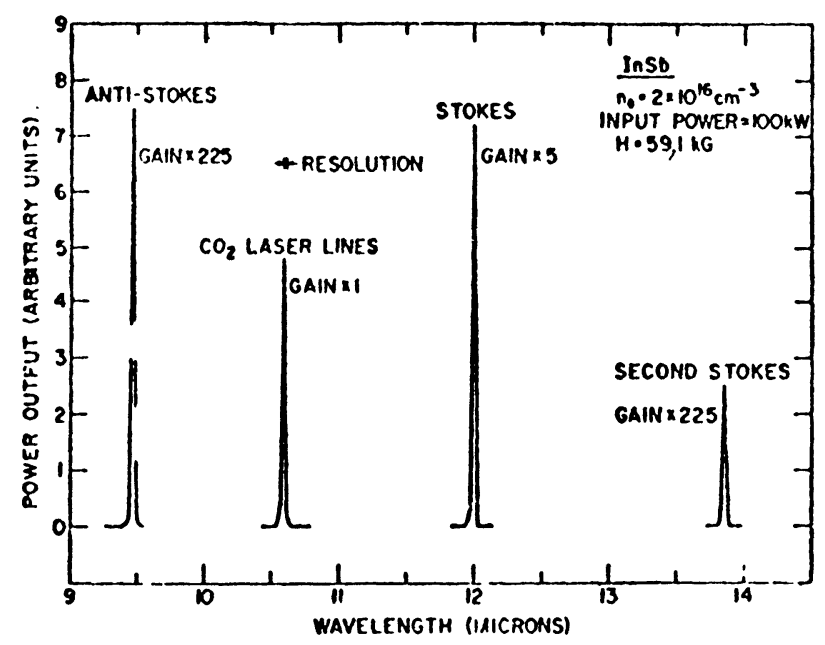

FIG. 8. - Spectre de diffusion Raman spin-flip stimulée dans InSb pompé avec un laser $\mathrm{CO}_{2} \mathrm{TE}$ (Aggarwal et al. [18]).

La figure 8 montre un spectre de diffusion stimulée dans InSb pompé avec un laser $\mathrm{CO}_{2}$ excité transversalement et à une pression supérieure à la pression atmosphérique (puissance-crête de l'ordre de $1 \mathrm{MW}$, Aggarwal et al. [18]). Une première raie Stokes de $1 \mathrm{~kW}$ a pu être obtenue ainsi qu'une $2^{\mathrm{e}}$ Stokes et une anti-Stokes dont les puissances relatives sont indiquées sur la figure. En pompant avec un laser CO continu, Patel [17] a pu observer jusqu'à la $4^{\mathrm{e}}$ Stokes (Fig. 9) et Mooradian [19] la $5^{\mathrm{e}}$ Stokes et la $2^{\mathrm{e}}$ anti-Stokes.
- FREAUEF:CY $\left(\mathrm{cm}^{-1}\right)$

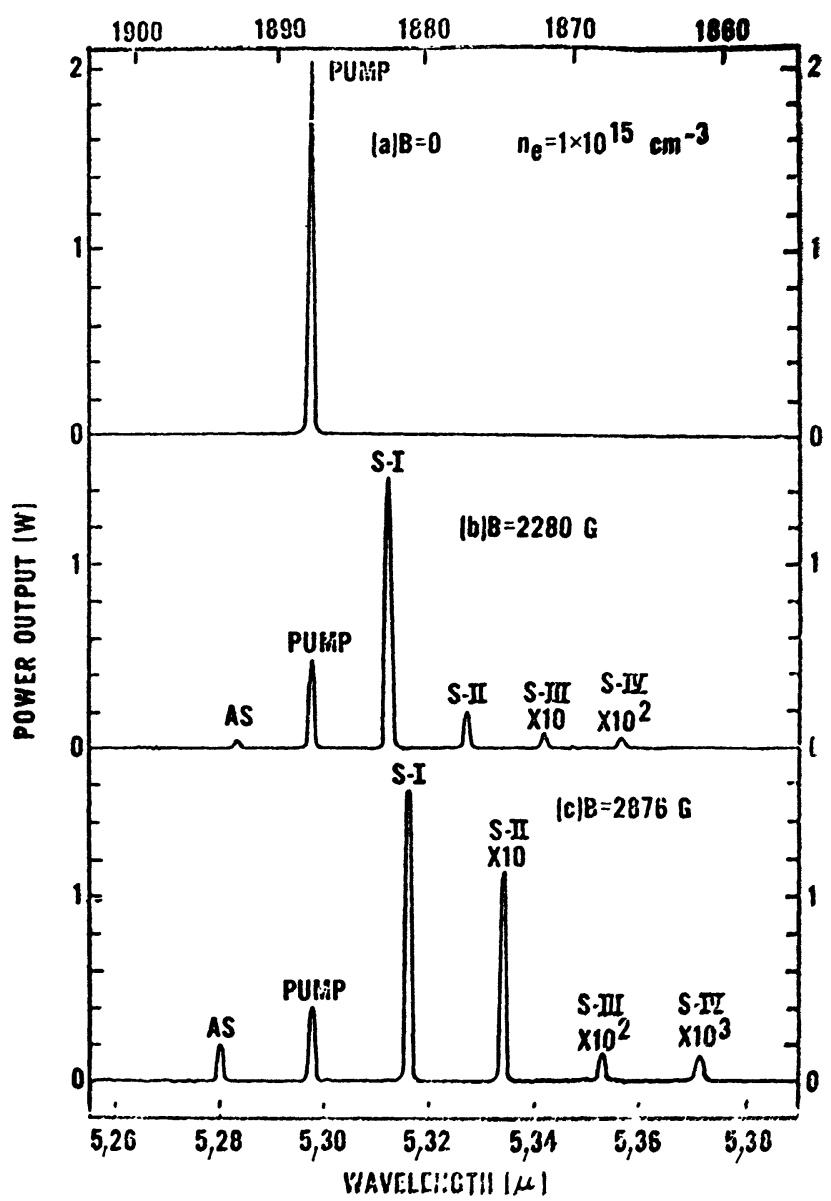

Fig. 9. - Spectre de diffusion Raman spin-flip stimulée dans InSb pompé avec un laser CO continu (Patel et al. [17]).

4.7 GAMmeS DE LONGUEUR D'ONDE ACCORDABLES. Les figures 10 et 11 donnent les gammes de longueurs d'onde accordables correspondant aux deux figures

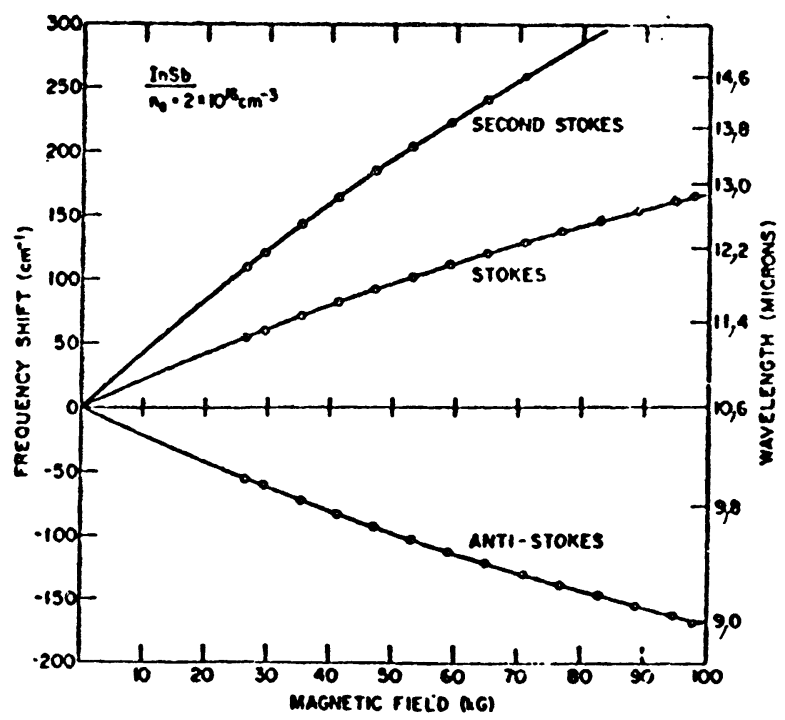

FIG. 10. - Gamme de longueur d'onde accordable autour de $10,6 \mu \mathrm{m}$ à partir des raies Stokes, anti-Stokes et second Stokes (Aggarwal et al. [18]). 
précédentes, c'est-à-dire autour de $10,6 \mu$ et autour de 5,3 $\mu$. Dans le premier cas, l'absence de points expérimentaux pour des champs magnétiques inférieurs à $26 \mathrm{kG}$ provient de ce que, pour un échantillon de $2 \times 10^{16}$ électrons par $\mathrm{cm}^{3}$, la condition de limite quantique n'est plus satisfaite. Dans le $2^{\mathrm{e}}$ cas, l'échantillon est dopé seulement à $1 \times 10^{15} \mathrm{~cm}^{-3}$, ce qui explique qu'on puisse descendre jusqu'à un champ magnétique de $600 \mathrm{G}$.

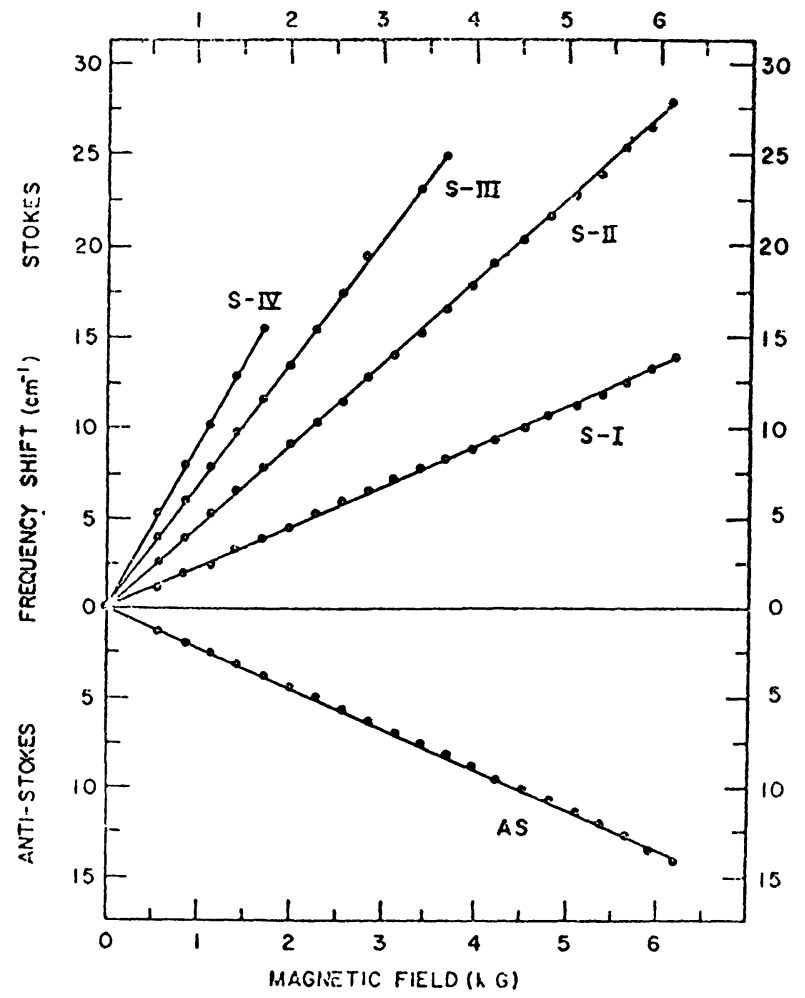

Fig. 11. - Gamme de longueur d'onde accordable autour de $5,3 \mu$, à faible champ magnétique, à partir des raies Stokes I, II, III, IV et de la raie anti-Stokes (Patel et al. [17]).

La figure 11 constitue en fait une étude à faible champ. Il est possible d'étendre la gamme accordable autour de 5,3 $\mu$ en prenant des champs plus élevés [24], ou encore en utilisant plusieurs raies du même laserpompe, ce qui a l'avantage de ne pas nécessiter de champ magnétique intense. C'est ce qu'illustre la figure 12, pour différentes raies d'un laser $\mathrm{CO}_{2}$ (TEA), doublé en fréquence [25].

4.8 LARGEUR DE RAIE. - La largeur de raie correspondant au processus spin-flip spontané est trop fine pour pouvoir être mesurée d'une manière conventionnelle en analysant le spectre de diffusion à l'aide d'un spectromètre. On utilise plutôt une technique dite d'amplification spin-flip. Deux faisceaux lumineux de fréquences fixes $\omega_{0}$ et $\omega_{s}$ traversent l'échantillon de InSb dans une certaine configuration. La plus faible fréquence, $\omega_{\mathrm{s}}$ correspondant à la Stokes, est amplifiée d'une manière résonnante lorsque le champ magnétique atteint la valeur donnée par $\omega_{0}-\omega_{\mathrm{s}}=\omega_{\mathrm{sf}}$.

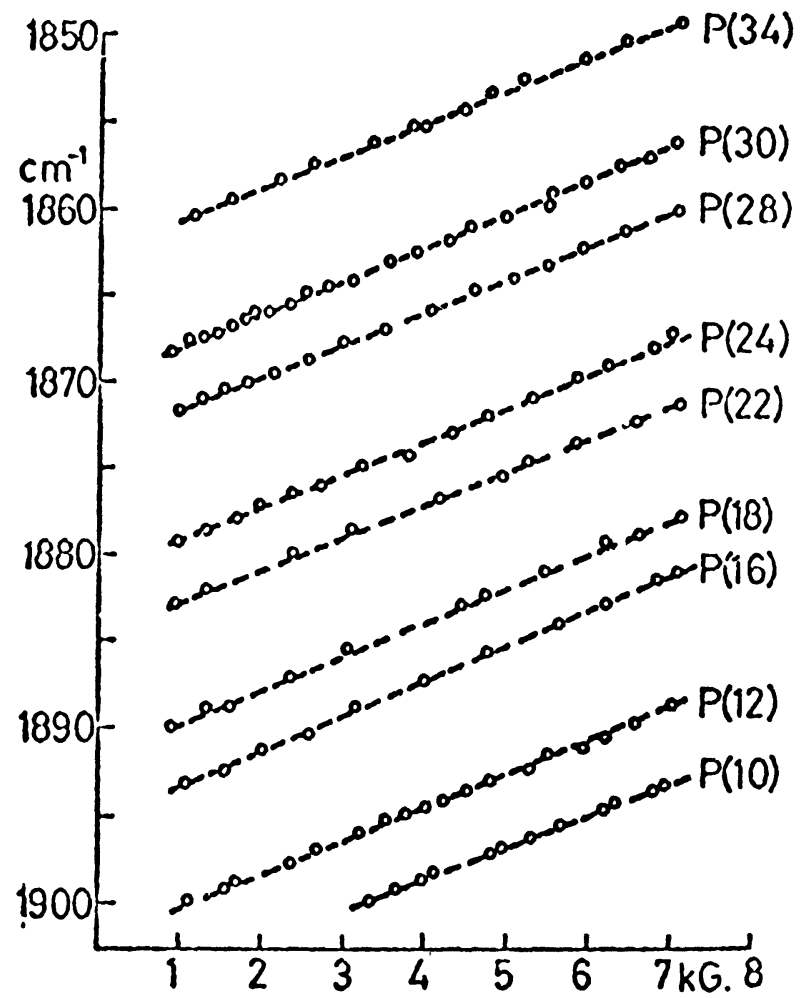

FIG. 12. - Gammes de longueur d'onde accordable pour différentes raies d'un laser $\mathrm{CO}_{2}$ TEA, doublé en fréquence (Smith et al. [25]).

En défilant en champ magnétique, si celui-ci est contrôlé avec une bonne précision, on peut avoir la variation du gain Raman avec une grande résolution, ce qui permet d'en déduire la largeur de raie $\Gamma_{\mathrm{S}}$ de la diffusion spontanée. Les valeurs trouvées sont de l'ordre de $200 \mathrm{MHz}$ [17], [19].

En fait $\Gamma_{\mathrm{S}}$ dépend de plusieurs facteurs comme le nombre de porteurs ou la température. Il a été observé [17] que $\Gamma_{\mathrm{S}}$ diminue d'un facteur 20 quand on réduit la concentration des porteurs d'un facteur 10 . D'autre part $\Gamma_{\mathrm{S}}$ a tendance à augmenter avec la température [29], ce qui explique que l'effet stimulé n'ait pas pu être observé au-delà d'une température de $\simeq 45 \mathrm{~K}$.

Quant à la largeur de raie de l'effet stimulé, elle a été mesurée directement par Patel [26] en utilisant une méthode hétérodyne. La valeur trouvée pour un laser spin-flip opérant à $5,3 \mu$ est $\Gamma_{\text {stim }}<1 \mathrm{kHz}$, qui est certainement la largeur de raie la plus fine qu'on connaisse pour une source cohérente accordable dans l'infrarouge.

4.9 Modes DE CAVITÉ. - Un facteur particulièrement important pour les applications en spectroscopie est le réglage fin de la longueur d'onde. La figure 13 montre le comportement de la raie Stokes à $5,3 \mu(\mathrm{CW})$ pour un déroulement fin en champ magnétique [27]. Dans la région de saturation, le signal est relativement stable, mais près du seuil, on obtient des oscillations qui traduisent un couplage entre la raie spin-flip et les modes de cavité. 


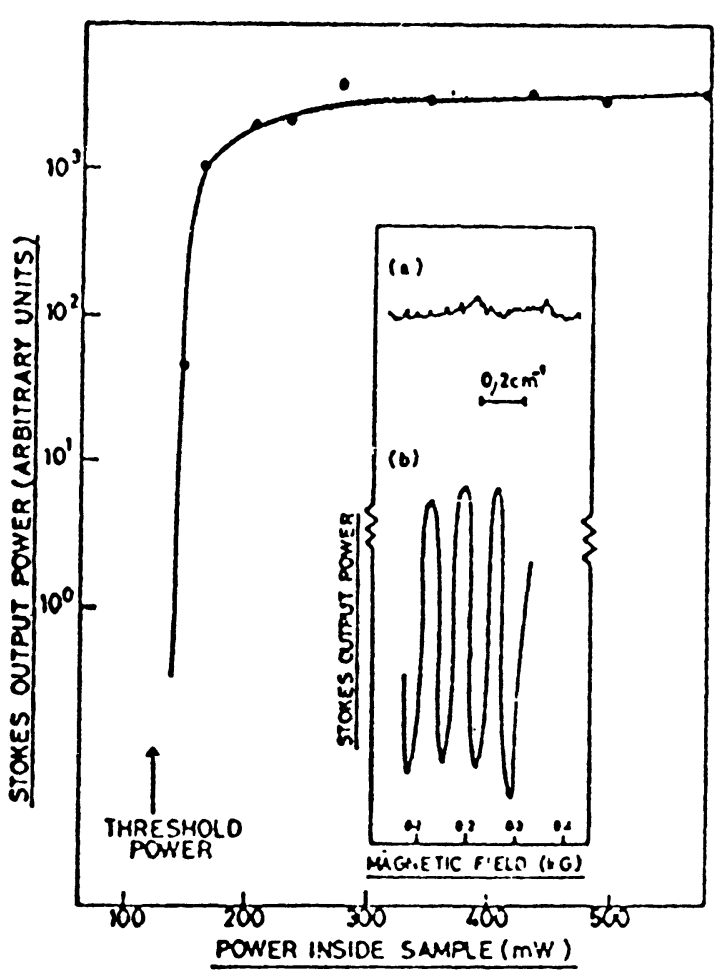

Fig. 13. - Comportement de la raie Stokes en fonction du champ magnétique $: a$ ) en région de saturation, $b$ ) près du seuil (Colles et al. [27]).

Brueck [28] a montré l'intérêt qu'on peut avoir à utiliser un laser spin-flip à faible concentration de porteurs et à faible champ magnétique. Un échantillon avec $N=10^{15} \mathrm{~cm}^{-3}$, à $4 \mathrm{kG}$, conduit à un taux de réglage de $60 \mathrm{MHz} / \mathrm{G}$ et des sauts de modes de $200 \mathrm{MHz}$, alors qu'un échantillon avec $N=10^{16} \mathrm{~cm}^{-3}$, à $40 \mathrm{kG}$, donne un taux de $16 \mathrm{MHz} / \mathrm{G}$ et des sauts de $6 \mathrm{GHz}\left(0,2 \mathrm{~cm}^{-1}\right)$.

En définitive, les échantillons de $\mathrm{InSb}$ à faible concentration de porteurs $\left(N=10^{15} \mathrm{~cm}^{-3}\right)$ semblent constituer actuellement les meilleurs lasers spin-flip. Ils ont l'avantage d'avoir un seuil de stimulation plus faible et une largeur de raie plus fine, ils permettent un réglage plus fin de la longueur d'onde, et enfin ils ne nécessitent pas de champ magnétique intense.

4.10 Nos PROPRES RÉSULTATS. - Nos mesures ont porté d'une part sur $\mathrm{CdS}$ pompé avec un laser à argon et d'autre part sur InSb pompé avec un laser à $\mathrm{CO}_{2}$, éventuellement doublé en fréquence par du Te.

Le CdS a un gap de $2,58 \mathrm{eV}$ à $4 \mathrm{~K}$. Il est donc transparent pour la raie $5145 \AA$ du laser à argon, cette longueur d'onde étant d'ailleurs très proche de sa limite d'absorption, ce qui permet de tirer profit de l'effet de résonance. Toutefois le facteur $g$ des électrons libres dans ce matériau est seulement de 1,76 (comparé à 50 dans InSb). Pour des champs magnétiques de l'ordre de $50 \mathrm{kG}$, les raies Stokes et anti-Stokes sont distantes de $4 \mathrm{~cm}^{-1}$ seulement de la raie Rayleigh, ce qui gêne leur observation.

Nous avons pu isoler ces raies grâce à l'utilisation

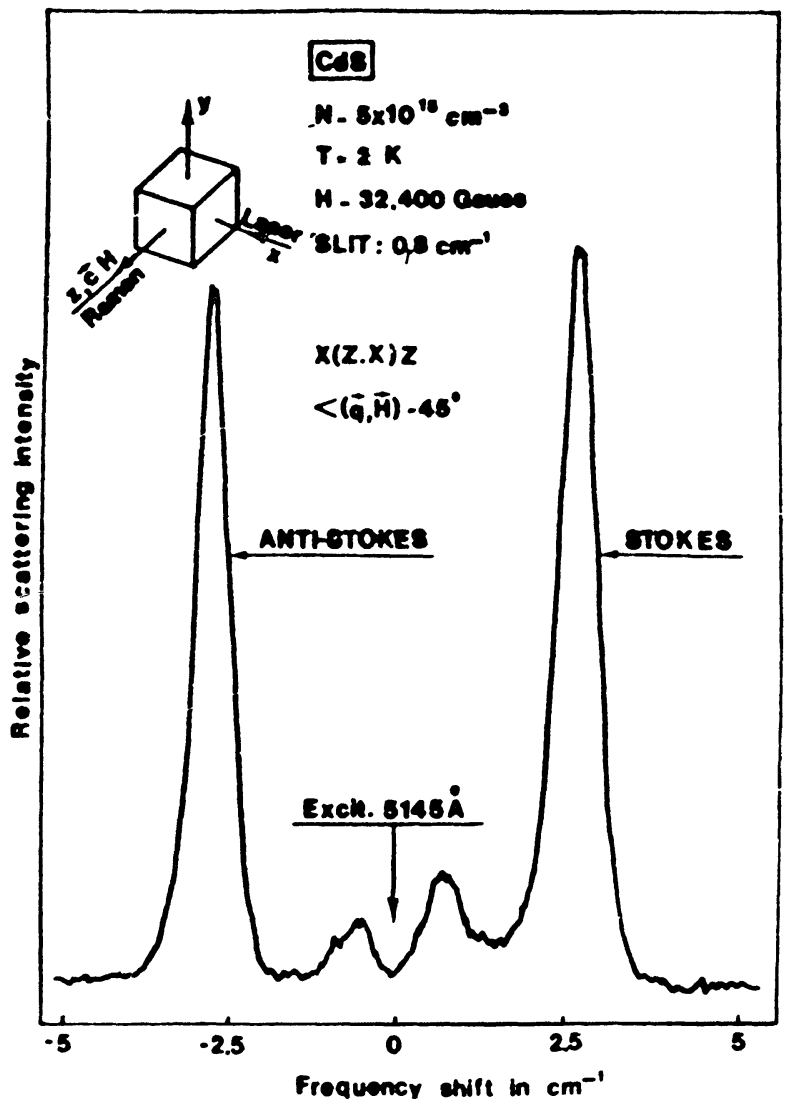

Fig. 14. - Diffusion Raman spin-flip dans CdS. La diffusion Rayleigh est éliminée par absorption dans $\mathrm{I}_{2}$ (Amzallag et al. [30]).

d'une cuve à iode placée dans le faisceau diffusé, qui absorbe et élimine complètement la lumière parasite à la fréquence excitatrice [30] (Fig. 14). Il s'agit là d'un effet de diffusion spontanée puisque la puissance de notre laser n'était que de $200 \mathrm{~mW}$, et que le seuil de stimulation est supérieur à $1 \mathrm{MW} / \mathrm{cm}^{2}$ [31]. Ces mesures nous ont permis de déterminer une valeur précise du facteur $g$, en bon accord avec les résultats d'autres techniques. Par ailleurs, utilisant la raie Stokes comme une source à longueur d'onde variable, c'est-à-dire en défilant en champ magnétique, nous avons pu relever un spectre d'absorption à haute résolution de l'iode, au voisinage de $5145 \AA$. La structure obtenue permet d'assurer que la largeur de la raie Stokes est inférieure à $600 \mathrm{MHz}\left(0,02 \mathrm{~cm}^{-1}\right)$.

Nos mesures sur InSb, relativement récentes, confirment certains des principaux résultats exposés précédemment, notamment la variation et la limitation de l'effet stimulé en fonction du champ magnétique, les gammes de fréquence accordables, etc... En fait, ces mesures sur InSb servent surtout de test pour notre dispositif expérimental, le but recherché étant l'observation et l'étude de la diffusion spin-flip dans les mélanges du type $\mathrm{Hg}_{1-x} \mathrm{Cd}_{x} \mathrm{Te}$ et $\mathrm{Pb}_{x} \mathrm{Sn}_{1-x} \mathrm{Te}$. Ces matériaux ont des facteurs $g$ élevés et surtout des gaps qui varient continûment avec la composition des mélanges, ce qui devrait étendre la technique des lasers spin-flip (CW) à d'autres gammes de fréquences infra- 
rouges. La qualité de ces matériaux liée à leur homogénéité était jusqu'ici insuffisante, elle a tendance à s'améliorer [32].

5. Applications actuelles. - Les applications les plus remarquables du laser spin-flip à l'heure actuelle se rapportent à la spectroscopie à haute résolution et à l'optique non linéaire.

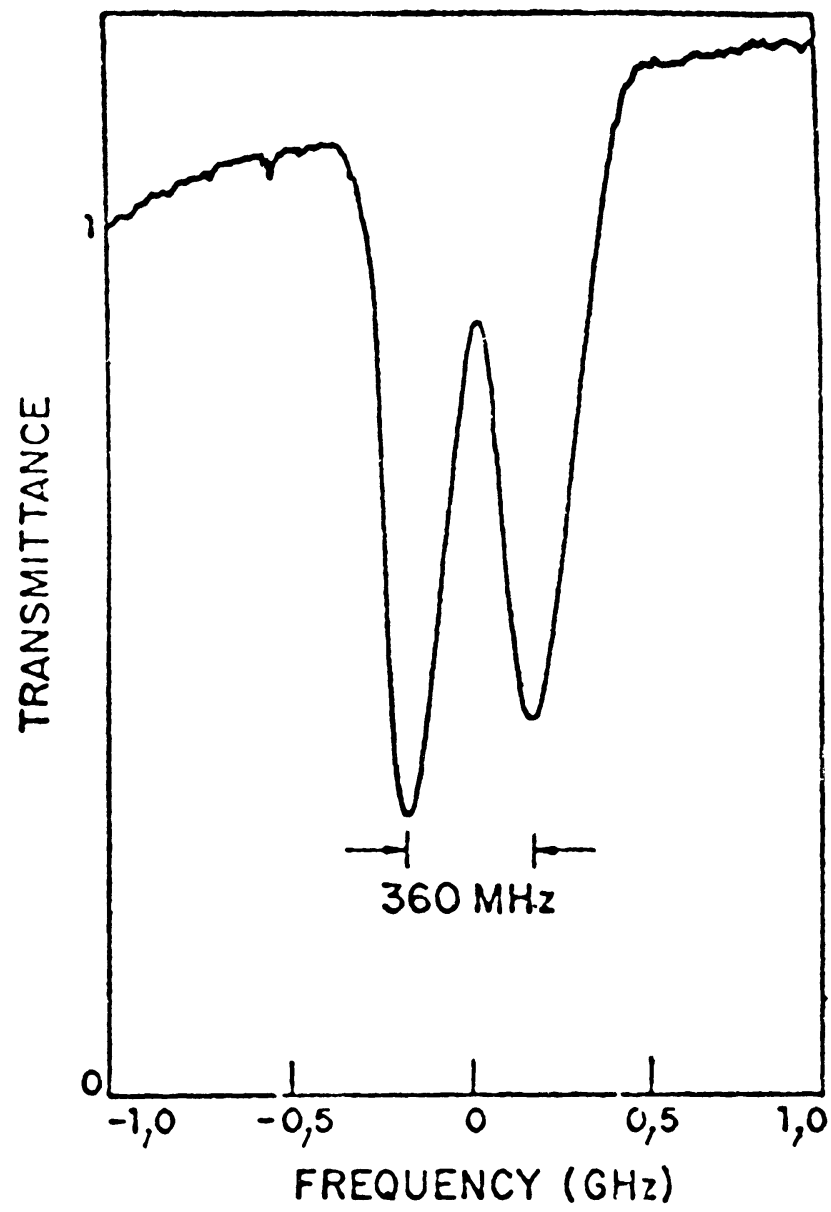

Fig. 15. - Spectre d'absorption de la raie $\mathbf{R}_{1 / 2}\left(\frac{1}{2}\right)$ de NO à $1881 \mathrm{~cm}^{-1}$ (Brueck et al. [28]).

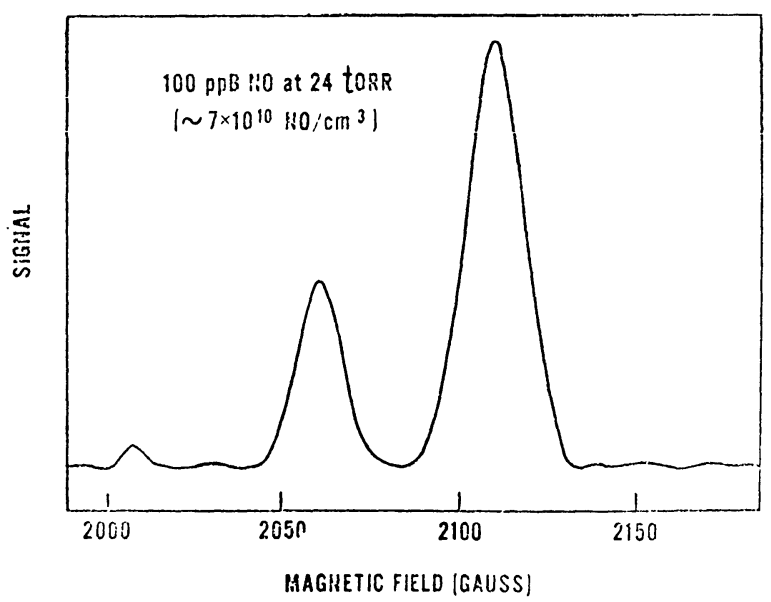

Fig. 16. - Spectre d'absorption de 0,1 partie par million de NO dans $\mathrm{N}_{2}$ à 24 torrs (Patel et al. [17]).
On peut illustrer les performances spectroscopiques du laser spin-flip à faible champ magnétique à l'aide des figures 15 et 16 . La $1^{\text {re }}$ figure représente le spectre d'absorption de NO à $1881 \mathrm{~cm}^{-1}$, où la raie $R_{1 / 2}\left(\frac{1}{2}\right)$ est parfaitement résolue [28], l'écart entre les deux raies composantes étant de $360 \mathrm{MHz}$. La figure 16 montre l'utilité que peut avoir le laser spin-flip dans la détection de la pollution de l'atmosphère. Patel [17] a utilisé le laser spin-flip avec une technique de détection acousto-optique (calorimétrique) pour mesurer de faibles teneurs de NO dans $\mathrm{N}_{2}$. Le spectre d'absorption de la figure correspond à 0,1 partie par million (24 torrs). La méthode permet donc de détecter une concentration de NO aussi faible que $7 \times 10^{8} \mathrm{~mol} / \mathrm{cm}^{3}$.

En optique non linéaire, le laser spin-flip peut être utilisé comme une source cohérente accordable pouvant être mélangée à un autre laser de fréquence fixe pour engendrer des fréquences somme ou différence, elles-mêmes accordables dans d'autres gammes de longueur d'onde. C'est ainsi que Pidgeon et al. [33] ont pu mélanger un laser spin-flip accordable entre 11,2 et $12,8 \mu$ et un laser $\mathrm{CO}_{2}$ (TEA), dans du Te orienté suivant l'accord de phase, pour générer une radiation stimulée à la fréquence somme, accordable entre 5,5 et $5,8 \mu$, ayant une puissance de l'ordre de $3 \mathrm{~W}$.

$\mathrm{Au}$ lieu de se servir de InSb comme source, on peut l'utiliser comme milieu mélangeur de deux fréquences incidentes et compter sur la non-linéarité optique liée à la résonance de spin pour intensifier la génération de la fréquence différence. Cela a été réalisé par Nguyen et al. [34] qui ont pompé un échantillon de InSb avec 2 lasers à $\mathrm{CO}_{2}$ opérant à $9,6 \mu\left(\omega_{1}\right)$ et $10,6 \mu\left(\omega_{2}\right)$ polarisés perpendiculairement. Lorsqu'on défile en champ magnétique, la puissance générée à la fréquence différence $\omega_{3}=\omega_{1}-\omega_{2}$ passe par une résonance aiguë lorsque $\omega_{3}=\omega_{\text {sf }}$ (Fig. 17). Cette technique est à rapprocher de celle de l'amplification de $\omega_{2}$ [§ 4.8] utilisée pour mesurer la largeur de la raie Stokes spontanée. En prenant un laser spin-flip comme l'une des deux sources, c'est-à-dire en combinant les processus de diffusion Raman spin-flip et de mélange par réso-

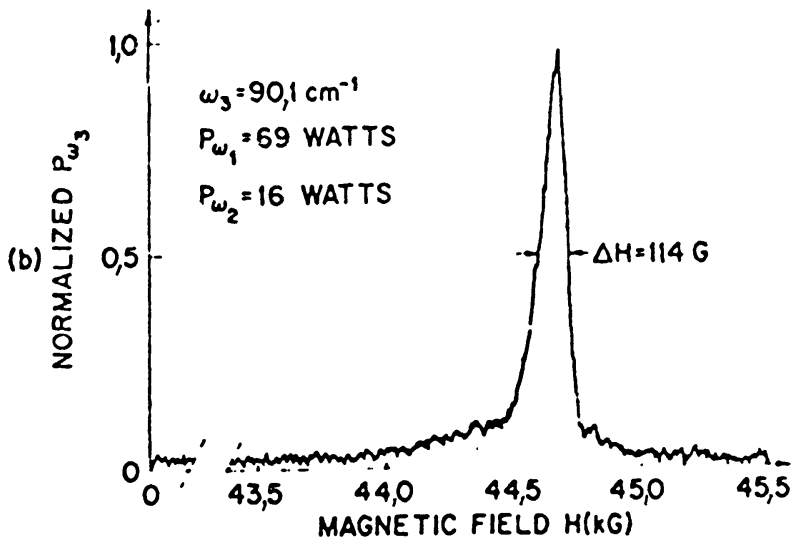

FIG. 17. - Génération d'une émission dans l'infrarouge lointain par différence de deux fréquences dans InSb (Van Tran et al. [34]). 
nance de spin, il a été possible de générer la fréquence différence qui correspond à de l'infrarouge lointain, et qui est accordable entre 93 et $100 \mathrm{~cm}^{-1}$ [34].

6. Conclusion. - La diffusion Raman spin-flip, caractérisée par une section efficace très élevée et une largeur de raie excessivement fine, constitue un outil puissant pour l'étude des propriétés physiques des électrons de conduction dans les semiconducteurs. Son extension à de nouveaux matériaux et ses applications en optique non linéaire devraient fournir de nouvelles sources cohérentes, accordables dans d'autres gammes de longueurs d'onde que celles qui sont exploitées actuellement.

\section{Bibliographie}

[1] BYer, R. L., Treatise in Quantum Electronics (éd. H. Rabin, N. Y.) 1974

[2] Kleinman, D. A., Boyd, G. D., J. Appl. Phys. 40 (1969) 546. [3] Yyarborough, J. M., Sussman, S. S., Puthoff, H. E., Pantell R. H., Johnson, B. C., Appl. Phys. Lett. 15 (1969) 102.

Amzallag, E., Chang, T. S., Johnson, B. C., Pantell, R. H., Puthoff, H. E., J. Appl. Phys. 42 (1971) 3251

[4] Heitler, W., Quantum theory of radiation (3rd ed Oxford Univ. Press) 1954.

[5] Dennis, R. B., Pidgeon, C. R., Smith, S. D., WherRetT, B. S. et Wood, R. A., Proc. R. Soc. A 331 (1972) 203.

[6] Bloembergen, N. et Shen, Y. R., Phys. Rev. 133A (1964) 37.

[7] Yafet, Y., Phys. Rev. 152 (1966) 858.

[8] WolfF, P. A., Colloque sur les semiconducteurs à faible gap. Nice. Sept. 1973 (à paraître).

[9] Wherret, B. S. et Harper, P. G., Phys. Rev. 183 (1969) 692.

[10] Brueck, S. R. J., Mooradian, A., Blum, F. A., Phys. Rev. B 7 (1973) 5253 ;

Yuen, S. Y., Wolff, P. A., LaX, B., Phys. Rev. B 9 (1974) 3394.

[11] Patel, C. K. N. et Shaw, E. D., Phys. Rev. B 3 (1971) 1279.

[12] Dennis, R. B., Wood, R. A., Pidgeon, C. R., Smith, S. D. et Sмith, J. W., J. Phys. C. Solid St. Phys. 5L (1972) 73.

WaChernig, H. et Grisar, R., Solid State Commun. 15 (1974) 1365.

[13] Wolff, P. A., Phys. Rev. Lett. 16 (1966) 225.

[14] Slusher, R. E., Patel, C. K. N., Fleury, P. A., Phys. Rev. Lett. 18 (1967) 77.

[15] Patel, C. K. N., Shaw, E. D., Phys. Rev. Lett. 24 (1970) 451.

[16] Mooradian, A., Brueck, S. R. J., Blum, F. A., Appl. Phys. Lett. 17 (1970) 481.

[17] Patel, C. K. N., Laser Spectroscopy Conf. Vail, Colorado 1973 .
[18] Aggarwal, R. L., Lax, B., Chase, C. E., Pidgeon, C. R., Limbert, D., Brown, F., Appl. Phys. Lett. 18 (1971) 383.

[19] Mooradian, A., Laser Spectroscopy Conf. Vail, Colorado 1973.

[20] Wherret, B. S., Firth, W. J., IEEE J. Quantum Electron. 8 (1972) 865 .

[21] Patel, C. K. N., Appl. Phys. Lett. 19 (1971) 400.

[22] Brueck, S. R. J., Mooradian, A., Phys. Rev. Lett. 28 (1972) 161.

[23] Smith, S. D., Allwood, R. L., Dennis, R. B., Firth, W. J., Wherrett, B. S., Wood, R. A., Hafele, H., Conf. Intern. Diffusion de la lumière Paris 1971 p. 187.

[24] Brueck, S. R. J., Mooradian, A., Appl. Phys. Lett. 18 (1971) 229.

[25] Smith, S. D., Pidgeon, C. R., Wood, R. A., McNeish, A., Brignall, N., Laser Spectroscopy Conf. Vail, Colorado 1973.

[26] Patel, C. K. N., Phys. Rev. Lett. 28 (1972) 649.

[27] Colles, M. J., Dennis, R. B., Smith, J. W., Webb, J. S., Opt. Commun. 10 (1974) 145.

[28] BRUECK, S. R. J., $12^{\mathrm{e}}$ Conf. Intern. Semiconduct. Stuttgart 1974, p. 780.

[29] Grisar, R. et Wachernig, H., $12^{\circ}$ Conf. Int. Semiconduct. Stuttgart 1974, p. 803.

[30] Amzallag, E., Dugautier, C., Moch, P., Balkanski, M., Solid State Commun. 12 (1973) 1303.

[31] Scott, J. F., Damen, T. C., Phys. Rev. Lett. 29 (1972) 107.

[32] Sattler, J. P., Weber, B. A., Nemarich, J., Appl. Phys. Lett. 25 (1974) 491.

[33] Pidgeon, C. R., Lax, B., Aggarwal, R. L., Chase, C. E., Appl. Phys. Lett. 19 (1971) 333.

[34] Nguyen, V. T., Bridges, T. J., Phys. Rev. Lett. 29 (1972) 359 ;

NguYen, V. T., Bridges, T. J., Laser Spec. Conf. Vail. Colorado 1973. 